\title{
LA DIGNIDAD COMO DERECHO HUMANO JUDICIALMENTE PROTEGIDO Y EL MODELO DE FAMILIA EN MÉXICO ${ }^{1}$
}

\author{
Walter Arellano Hobelsberger \\ Magistra do del Q uinto Tribunal Colegiado en \\ Material Civil \\ Primer Circ uito. Suprema Corte de J usticia de \\ la Nación (México)
}

\begin{abstract}
Resumen
El concepto de dignidad humana asentado en la Constitución mexicana vigente es un valor supremo que debe ser considerado principio fundamental informador de todo el ordenamiento jurídico. Cuando en el año en curso se planteó la posible contravención a la Carta Magna derivada del hecho de que la Asamblea Legislativa del Distrito Federal determino redefinir la institución del matrimonio para permitir que dos personas del mismo sexo puedan contraerlo y se instituyó la adopción para este tipo de matrimonios. Uno de los aspectos más sobresalientes de la resolución 7/2010 pronunciada por la Suprema Corte de Justicia de la Nación fue justamente el relativo al respeto de la dignidad de la persona humana sobre la base de la prevalencia del principio de igualdad entendido como un valor supremo.
\end{abstract}

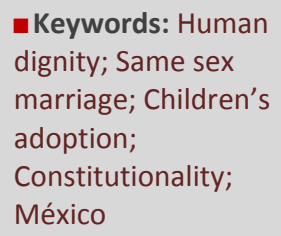

- Palabras clave: Dignidad humana; Matrimonio personas del mismo sexo; Adopción infantil; Constitucionalidad; México

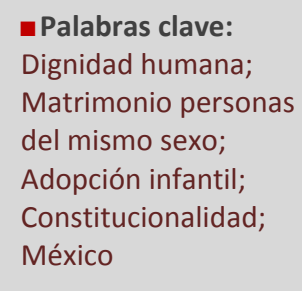

\begin{abstract}
Human dignity has been recognized as basic principle for the entire legal order in accordance with mexican Constitution of 2005. The Suprema Corte de Justicia de la Nación has explained the content of this principle when settled its judgment $7 / 2010$ about same sex marriage and adoption of children by these relationships.
\end{abstract}

${ }^{1}$ Conferencia ofrecida el día 16 de octubre de 2010 en la Sede del Instituto de Investigaciones Jurisprudenciales y de Promoción y Difusión de la Ética Judicial - México DF (México). La mesa en la que fue pronunciada esta conferencia fue presentada y moderada por el Prof. Dr. José Ramón Narváez Hernández, Profesor del Posgrado en Derecho de la Universidad Nacional Autónoma de México e Instituto de Investigaciones Jurisprudenciales y de Promoción y Difusión de la Ética Judicial de la Suprema Corte de Justicia de la Nación, representante del partner internacional en México y coordinador de la actividad en aquel país. 
Versión escrita de la conferencia ofrecida el 16 de octubre de 2010 en el marco de la "II Experiencia Interdisciplinar de Didáctica a Distancia. Familia y relaciones afectivas.

Derecho, ideología y dinámicas sociales"

Buenos días, saludo a todas las sedes a las que estamos conectados.

Quiero agradecer, en primer lugar, la invitación que recibí del Doctor Ramón Narváez Hernández para formar parte de estas interesantes Jornadas de Didáctica a Distancia. Asimismo es un honor formar parte de esta mesa junto con el Dr. Jorge Adame Goddard y, por supuesto, que en el mismo tenor de agradecimiento a los distinguidos estudiantes y especialistas jurídicos que en otras latitudes están comunicados con nosotros gracias al avance de la tecnología.

En los últimos años, el sistema jurídico mexicano ha experimentado cambios significativos en su configuración constitucional y legal sobre todo lo que hace a la interpretación y a la argumentación del Derecho.

Un elemento sumamente notable en la evolución jurídica mexicana es la inclusión de la fraseología "dignidad humana” en el texto de nuestra Constitución que recién se dio a partir de los primeros años del presente siglo. Nuestra Constitución mexicana establece en su primer artículo lo que se conoce en nuestro medio jurídico como "garantía de igualdad" a través de la cual se prohíbe expresamente toda discriminación motivada por origen étnico, o nacional o, por supuesto, el género, la edad, las discapacidades, la condición social, la religión, las opiniones, las preferencias, el estado civil o cualquier otra que atente contra la dignidad humana y tenga por objeto anular o menoscabar los derechos y libertades de las personas.

Nótese que se habla de "las preferencias" y se habla también de "cualquier otra que atente contra la dignidad humana y tenga por objeto anular o menoscabar los derechos y libertades de las personas”.

Me parece que son muchas las lecturas que se le pueden dar a ese nuevo contenido del artículo primero de nuestra Carta Magna en relación con lo que debe entenderse por la frase “dignidad humana”. Dicho precepto se encuentra previsto en el capítulo que se conoce como “de garantías individuales” y, por tanto, puede pensarse que no se ha incorporado a nuestro Texto Fundamental una concepción plena de un derecho humano en toda su extensión. Sin embargo, desde una perspectiva diversa, también puede leerse el indicado numeral con una visión más extensa y optimista en el sentido de considerar que la inclusión de la dignidad humana en nuestra Constitución significa la pauta más importante que ha proporcionado el constituyente reformador para recordarnos que la dignidad humana es un valor supremo que debe reconocerse como prioritario en el sistema jurídico mexicano.

En lo personal, más allá de la postura de la que nos afiliemos, me parece que esa sola inclusión expresa que la dignidad humana en nuestro Documento Fundamental 
es la más importante de las múltiples reformas que ha experimentado desde su configuración en el año de 1917.

Estoy convencido de que para descifrar lo que debe entenderse por dignidad humana hemos de acudir al trabajo que han desarrollado distinguidos filósofos en distintos momentos de la historia del mundo, pues no hemos sido los abogados los más hábiles en desempeñar esa tarea, como tampoco lo hemos sido cuando ha sido el momento de delimitar lo que entendemos por Justicia.

Trataré de explicar las razones por las cuales se hace esta afirmación.

Para caracterizar conceptualmente la dignidad humana como se desprende de las reflexiones de ilustres pensadores de épocas diversas pero, al fin, filósofos, se podría proponer la siguiente exégesis de lo que ha sido la dignidad.

Se habla de dignidad como aquello que es valioso, que está revestido de excelencia, o de realce. Dignidad humana equivale a la dignidad de la persona. Es un atributo de los animales superiores. Es un ente importante en el Universo. Cuando se habla de dignidad se alude a que la persona humana es lo más digno de la naturaleza. La dignidad pertenece a la esencia. Es la bondad de alguna cosa por causa de sí misma. La dignidad se descubre con la razón. La dignidad en los seres humanos nos remite a algo, además de valioso, inviolable. No entra en el campo de lo negociable. No requiere ser reconocido, solo existe, pero debe descubrirse. Lo valioso se refiere a las perfecciones del ser en sí mismo y ahí radica lo bueno de sí mismo desde el punto de vista ontológico.

En la antigua China y también en Roma, la dignidad se concibe como una idea universal que permite reflejarse como la igualdad esencial de todos los hombres. Aristóteles, lo sabemos todos, consideraba que el ser humano es un ser político. Todo hombre, por su naturaleza, es miembro de la comunidad del género humano gobernado por la Razón y, además, miembro de la propia comunidad política en la cual nace. La Escuela Estoica, que desarrolló el pensamiento aristotélico, llegó a la conclusión de que el hombre por su naturaleza es miembro de la comunión universal del género humano; la dignidad es el resultado del buen equilibrio emocional, libertad, inteligencia, buena decisión.

La dignidad es un valor intrínseco y superior de cada ser humano independientemente de su posición social, cultural y económica. La dignidad es el pilar de todos los derechos humanos. La dignidad no es un derecho propiamente, es la base de todos los derechos. La dignidad se basa en el reconocimiento de que la persona merece respeto.

La referencia a la dignidad humana es omnipresente en los instrumentos fundacionales del Derecho internacional de los derechos humanos concluida la Segunda Guerra Mundial: Con la Declaración Universal de los Derechos Humanos de 1948, en cuyo preámbulo refiere la dignidad intrínseca de todos los miembros de la familia humana y luego refiere "todos los seres humanos nacen libres e iguales en dignidad y derechos”. 
La preocupación hoy por la persona es un referente universal. La universalización o globalización de la dignidad es un presupuesto para la consecución de una verdadera emancipación y pacificación de la humanidad.

Inmanuel Kant, en su segunda fórmula del imperativo categórico decía: “Obra tanto en tu persona como en la persona del otro siempre como un fin, nunca como un medio". Es decir, no puede ser sustituido por otro, no tiene precio. Para Kant, solamente el hombre y la dignidad son términos absolutos. Desde una perspectiva ética y filosófica todo se basa en la 'otredad', en reconocer a otro ser humano en sus derechos.

Todo este bagaje de expresiones, digamos, de carácter filosófico nos conduce a constatar la importancia de la dignidad humana que, aunque tardíamente llega a México, constituye actualmente un pilar fundamental en nuestro sistema constitucional.

Convencido de la importancia de fijar una delimitación conceptual, que sirva a los tribunales, sobre qué debe entenderse por dignidad humana, de conformidad con la actual redacción de la Constitución Política de los Estados Unidos Mexicanos, en el mes de julio de este año, con motivo de un asunto del orden familiar que versó sobre el derecho de visitas y convivencias respecto de menores, propuse al Pleno del Tribunal al que pertenezco, que aceptaron, dos tesis interpretativas que en breve formarán jurisprudencia de aplicación obligatoria, sobre la definición, la naturaleza y el concepto, precisamente, de la dignidad humana. Tales interpretaciones están publicadas en la página 2273, del tomo 32, del Semanario Judicial de la Federación, correspondiente al mes de agosto del año en curso, en las que se establece textualmente lo siguiente: "Dignidad humana, definición. La dignidad humana es el origen, la esencia y el fin de todos los derechos humanos". Otra tesis: "Dignidad humana, su naturaleza y concepto. La dignidad humana es un valor supremo establecido en el artículo primero de la Constitución Política de los Estados Unidos Mexicanos en virtud del cual se reconoce una calidad única y excepcional a todo ser humano por el simple hecho de serlo, cuya plena eficacia debe ser respetada y protegida integralmente sin excepción alguna”.

Cada vez es mayor el número de asuntos en nuestro país en los que se hacen planteamientos de hecho y de Derecho que requieren enfocar los problemas desde la perspectiva de la dignidad humana.

Así, por ejemplo, este año se presentó ante la Suprema Corte de Justicia de la Nación, que configura un verdadero tribunal constitucional a partir del año 1995, una acción de inconstitucionalidad, consistente en un medio de control abstracto de la Constitución, en donde se planteó la posible contravención a la Carta Magna derivada del hecho de que la Asamblea Legislativa del Distrito Federal, que corresponde aquí, en México, a la Capital de la República, se determino redefinir la institución del matrimonio para permitir que dos personas del mismo sexo puedan contraer matrimonio y, además, se legisló para facilitar la institución familiar de la adopción para este tipo de matrimonios. 
La Suprema Corte resolvió declarar la validez de los artículos que permiten el matrimonio entre personas del mismo sexo y la adopción de menores por esta nueva forma matrimonial. Bien se puede decir que ante una fuerte oposición social ante una temática tan polémica, encabezada especialmente por sectores de la Iglesia, se trató de una decisión que de cierta forma pudiera ser calificada como democrática pero antipopular. Lo que no significa que en modo alguno exista contradicción en ello pues la Corte se ciñó a los principios y valores constitucionales aunque las estadísticas que en su oportunidad se conocieron no estaban de acuerdo con la decisión jurisdiccional del Máximo Tribunal. También se pudiera decir que la intervención o, en su caso, la interpretación de la Corte, no siguió los parámetros del Sr. Ronald Dworkin en cuanto al señalamiento que hace en sus obras en el sentido de que debe interpretarse la Constitución conforme a la cultura, la tradición y las costumbres.

Uno de los aspectos más sobresalientes de la resolución pronunciada por el Tribunal Constitucional fue justamente el relativo al respeto de la dignidad de la persona humana sobre la base de la prevalencia del principio de igualdad entendido como un valor supremo.

En el contexto esencial del fallo, se advierte la clara intención de establecer esquemas que permitan empapar la realidad social y el Derecho. Ver lo que quiere verse invisible y afrontar un compromiso con la institución de la familia.

Para ello, la Suprema Corte de Justicia de la Nación, es muy contundente en mencionar que la Constitución, particularmente en su artículo cuarto, protege la organización y el desarrollo de la familia en sus múltiples formas y manifestaciones. En ese sentido, se destaca que la familia, lejos de ser un concepto jurídico, es un concepto sociológico, que experimenta cambios y transformaciones de carácter social a lo largo del tiempo las cuales impactan sustancialmente en la estructura organizativa de la familia en cada época. De esta forma, los fenómenos de carácter social, como la incorporación cada vez más activa de la mujer al trabajo, el menor número de hijos, la tasa de divorcios, las nuevas nupcias que dan origen a familias integradas con hijos de matrimonios o de uniones anteriores e, inclusive, con hijos en común de los nuevos cónyuges, el aumento de madres y de padres solteros, las uniones libres o de hecho, la reproducción asistida, la disminución de la tasa de natalidad, la migración y la economía, entre otros muchos factores, han dado lugar a que la "familia tradicional", como se conoce en nuestro país, ha venido cambiando con el trayecto de los años.

Por ello es que, en principio, se justifica que el legislador ordinario se encuentre compelido a enfrentar esa realidad social y en eso también está comprometido el Tribunal Constitucional Mexicano. Se acepta que, tradicionalmente, está reconocido que el matrimonio como institución civil lo celebra un hombre y una mujer y es la base primaria de la familia y, como tal, ha sido objeto de especial protección por parte del Estado. Sin embargo, debe reconocerse, así lo dice la resolución, también que el matrimonio no debe ser un concepto petrificado o inmutable pues las relaciones sociales han presentado transformaciones de gran envergadura en las que se involucran las personas y las familias. En ese entorno evolutivo, se ha 
flexibilizado el divorcio, se ha creado el concubinato así como las sociedades de convivencia y, al mismo tiempo, en ese proceso de cambio, se ha ido separando la idea, antes intocada, de matrimonio - procreación que, en algún momento, fue importante y casi un axioma.

Aunque el Estado, ciertamente, está interesado en la protección de la familia de la que no escapa una intención excepcional en su protección constitucional, no debe hablarse de que solamente debe de protegerse a la denominada "familia ideal", formada por el padre la madre y los hijos, pues ello sería precisamente atentar contra la dignidad humana de quienes no forman parte de ese tipo de familia que, por cierto, cada vez constituyen un mayor número de personas, entre las que se encuentran, naturalmente, un número importante de menores de edad.

Bajo tales condiciones, aquí adquiere una gran relevancia la mención que se hace, a la luz de la dignidad humana, de los derechos de la personalidad que están en juego. Así, la decisión de un individuo de unirse a otro y proyectar una vida en común, como la relativa a tener hijos o no, deriva de la autodeterminación de cada persona, del derecho al libre desarrollo de la personalidad de cada individuo, que en diversas resoluciones ha reconocido la Suprema Corte de Justicia Mexicana.

En la resolución que comentamos, se asienta con toda claridad que la interpretación constitucional debe estar en correspondencia con la realidad social y debe buscar tutelar las múltiples diferencias que caracterizan a una sociedad asentada en la pluralidad y heterogeneidad de intereses, expectativas y preferencias y, especialmente, en el caso de la redefinición del matrimonio para incluir contrayentes del mismo sexo, se involucra una ampliación o bien, visto de otra forma, la igualdad de las personas homosexuales y la protección de sus uniones, que evidentemente han transitado dentro de la sociedad por un camino bastante difícil, de tal forma que, como ha sido reiterado en la jurisprudencia comparada, tanto en la interpretación como en la aplicación de los derechos fundamentales contenidas en los tratados internacionales, deben aplicarse en todo caso los principios pro persona y pro libertatis, conforme a los cuales siempre debe optarse por una interpretación extensiva en todo lo que favorezca su ejercicio y restrictiva en todo lo que los limite o se contraponga a dicha libertad.

Todo ello lleva al Tribunal Constitucional a considerar que la nueva definición del matrimonio en México constituye una decisión legislativa que es constitucionalmente razonable y proporcional, de tal forma que se trata de preservar la dignidad humana como un derecho fundamental, superior, reconociendo el sistema jurídico mexicano que deriva del libre desarrollo de la personalidad, es decir, del derecho de todo individuo a elegir en forma libre y autónoma cómo quiere vivir su vida, lo que comprende, entre otras expresiones, la libertad de contraer matrimonio o no hacerlo; la de procrear hijos, a decidir cuántos o bien decidir no tenerlos; la de escoger su apariencia personal y también, por supuesto, su libre opción sexual.

En otro orden de ideas, los lazos afectivos o sexuales, son derechos de la personalidad, por lo que es correcto que se generen condiciones de plena igualdad para todos los individuos, de tal manera que la inclusión del matrimonio, sin 
discriminación por razón de preferencias, también contempla relaciones de afectividad, sexualidad, solidaridad, estabilidad, permanencia y proyecciones de carácter común.

En cuestión de adopción de menores, igualmente, se reseña en la resolución respectiva que la protección del interés superior de los niños y las niñas que consagra el artículo cuarto de nuestra Constitución Federal es un principio que exige del Estado y de todos sus niveles de gobierno y ámbitos competenciales que siempre se procure su pleno respeto.

Es cierto que, tratándose de la institución civil de la adopción, los derechos de los menores sujetos a esta adopción se encuentran en posición prevalente frente al interés del adoptante o de sus adoptantes, dada precisamente esa protección constitucional excepcional de los niños y de las niñas. Sin embargo, ello no puede traducirse en que la orientación sexual de una persona o de una pareja, que es simplemente una de las opciones que se presentan en la naturaleza humana, y como tal forma parte de la autodeterminación y libre desarrollo de la personalidad, le reste valor como ser humano o pareja y, por tanto, lo degrade a considerarlo, por ese solo hecho, como nocivo para el desarrollo del menor y, por ende, que el legislador deba prohibir la adopción por parte de un matrimonio conformado por personas del mismo sexo o estimar que el solo hecho de que se trate de parejas del mismo sexo afecta al interés superior del menor.

Según se advierte de la multicitada resolución, no se tiene base normativa alguna para declarar inconstitucional el artículo relativo del Código civil para el Distrito Federal apoyándose en que la adopción por parejas del mismo sexo, per se, afecta el interés superior del menor y cualquier argumento en esa dirección nos pondría en la necesidad de utilizar un razonamiento vedado, precisamente, por el artículo primero de la Constitución Federal que específicamente prohíbe, como dijimos, la discriminación de las personas por razón de sus preferencias o por cualquier otra razón que atente contra la susodicha dignidad humana. Lo que también sería contrario a las diversas interpretaciones que ha desarrollado y confirmado el Alto Tribunal respecto del tipo de familia que protege el artículo cuarto de la Constitución y los derechos de los menores.

He de destacar que, para la Corte, pensar que la Constitución exige excluir del régimen legal que discipline la adopción a una categoría entera de personas definidas por su orientación sexual, implica caer en un razonamiento prohibido constitucionalmente. Asimismo, para el Tribunal Constitucional mexicano, no se observa qué diferencia constitucional o jurídica habría entre excluir a toda una categoría de personas del régimen legal de la adopción por motivos de orientación sexual o excluirla por motivos de raza, por ejemplo, o de origen étnico, o religioso, o económico; por citar, simplemente, algunos casos.

Por las mismas razones que no necesita conocerse el efecto que puede tener en los niños vivir en familias indígenas o no indígenas, familias pobres o familias ricas, familias con padres que tienen una discapacidad o no la tienen; porque en cualquier caso estaría constitucionalmente vedado no considerarlas una familia protegida por 
la Constitución o una familia, digamos, 'amenazante' -entre comillas- o 'disfuncional' -también entre comillas- para los niños. La Constitución hace que esa misma averiguación resulte verdaderamente innecesaria.

Según se afirma en la pluricitada resolución, en el caso de la adopción, lo que exige el principio del interés superior del menor es que la legislación aplicable permita delimitar el universo de posibles adoptantes sobre la base de que ofrezca las soluciones necesarias para el cuidado y desarrollo del menor, claramente establecidas en ley, y para que de esta forma la autoridad aplicadora evalúe y decida respecto de la que represente su mejor opción de vida.

Se dice así, en este sentido, que el Estado no está obligado a garantizar la supuesta situación de carácter utópico, y no jurídicamente asible, que la demanda en este caso de acción de inconstitucionalidad se invoca, cuando se habla de la necesidad de dar al adoptado "los mejores padres posibles". Si el Estado tuviera que garantizar este estándar de "los mejores padres posibles" el régimen de adopciones quedaría absolutamente inoperativo, por una parte, y, probablemente, también resultaría gravemente violatorio del párrafo tercero del artículo primero de la Constitución.

Incluso, según se asienta en el fallo relatado, en caso de que se considerara que ese estándar puede ser manejado dentro del contexto valorativo de nuestra Constitución, el mismo no permitiría excluir, por imperativo constitucional -como se pretende-, a toda una categoría de personas definida por una sola característica, como si fuera admisible constitucionalmente reducir a las personas a una sola de las características que tienen, que tenemos, todos como seres humanos.

De manera especial, el interés superior del menor exige que el Estado asegure que los niños se convertirán en adultos en contextos familiares que, prima facie, les garantizan cuidado, sustento y educación. Pero pensar que las familias integradas por personas del mismo sexo no satisfacen ese esquema implica caer un razonamiento constitucionalmente contrario a los intereses de los menores que, se afirma, se quieren proteger.

Según se ve también en esta resolución constitucional que se analiza, concluir que en estos casos la familia no satisface unas garantías de cuidado esperable de los niños que sea razonablemente alta, resulta insostenible dentro de la Constitución y particularmente contrario al Derecho fundamental, que tenemos todos los habitantes de este país, a no ser discriminados.

Es de ahí de donde se concluye que la Suprema Corte no puede suscribir de ningún modo que sea la preferencia o la orientación sexual de un ser humano el elemento utilizado o que sirva para que, a priori, se establezca que una persona o pareja de carácter homosexual puede tener la opción de adoptar un menor una vez que se han satisfecho, por supuesto, los requisitos y el procedimiento que al efecto establezca la legislación aplicable pues ello, sin duda alguna, se constituirá en una discriminación por orientación sexual que está expresamente proscrita por el artículo primero de nuestra Constitución, al basarse esta restricción o limitación exclusivamente en la preferencia sexual de una persona que, como ya señalamos anteriormente, en modo 
alguno debe afectar u obstaculizar el ejercicio de sus derechos fundamentales. Pero que, además, llevado al aspecto que ahora nos ocupa, tampoco puede verse como un elemento o factor que por sí mismo pudiera afectar el desarrollo de un menor.

Por ello es que la posibilidad jurídica de que matrimonios de un mismo sexo puedan realizar una adopción no debe considerarse, como no sucede tampoco con los heterosexuales, como una autorización automática e indiscriminada para adoptar sino que, obviamente, tiene que sujetarse al sistema legalmente establecido para tal efecto, en cuanto tiene como finalidad el aseguramiento del interés superior del menor como derecho fundamental del adoptado.

A manera de conclusión, se puede afirmar que la resolución de la Suprema Corte Mexicana ha dado un paso importante en la consolidación de una forma constitucional y democrática de asumir el fenómeno jurídico no vista antes en la historia de nuestro sistema jurídico mexicano.

Hasta aquí mi intervención. Por su atención, muchas gracias. 